\title{
Room-Temperature Hot-Polaron Photovoltaics in the Charge-Ordered State of a Layered Perovskite Oxide Heterojunction
}

\author{
B. Kressdorf, ${ }^{1}$ T. Meyer®, ${ }^{2}$ A. Belenchuk $₫,{ }^{3,4}$ O. Shapoval, ${ }^{3,4}$ M. ten Brink, ${ }^{5}$ S. Melles, ${ }^{1}$ U. Ross, ${ }^{1}$ \\ J. Hoffmann $\odot,{ }^{1}$ V. Moshnyaga, ${ }^{3}$ M. Seibt $\odot,{ }^{2}$ P. Blöchl, ${ }^{5,6}$ and C. Jooss ${ }^{1,}{ }^{1 *}$ \\ ${ }^{1}$ University of Goettingen, Institute of Materials Physics, Friedrich-Hund-Platz 1, 37077 Goettingen, Germany \\ ${ }^{2}$ University of Goettingen, 4th Physical Institute - Solids and Nanostructures, Friedrich-Hund-Platz 1, 37077 \\ Goettingen, Germany \\ ${ }^{3}$ University of Goettingen, 1st Physical Institute, Friedrich-Hund-Platz 1, 37077 Goettingen, Germany \\ ${ }^{4}$ IIEN, Academy of Sciences in Moldova, str. Academiei 3/3, MD-2028, Chisinau, Moldova \\ ${ }^{5}$ Institute for Theoretical Physics, University of Goettingen, Friedrich-Hund-Platz 1, 37077 Goettingen, Germany \\ ${ }^{6}$ Institute for Theoretical Physics, Clausthal University of Technology, Leibnizstr. 10, D-38678 \\ Clausthal-Zellerfeld, Germany
}

(Received 5 June 2020; revised 25 September 2020; accepted 9 October 2020; published 4 November 2020)

Harvesting of solar energy by hot carriers from optically induced intraband transitions offers new perspectives for photovoltaic energy conversion. Clearly, mechanisms slowing down hot-carrier thermalization constitute a fundamental core of such pathways of third-generation photovoltaics. The intriguing concept of hot polarons stabilized by long-range phonon correlations in charge-ordered strongly correlated three-dimensional metal-oxide perovskite films has emerged and been demonstrated for $\operatorname{Pr}_{0.7} \mathrm{Ca}_{0.3} \mathrm{MnO}_{3}$ at low temperature. In this work, a tailored approach to extending such processes to room temperature is presented. It consists of a specially designed epitaxial growth of two-dimensional Ruddlesden-Popper $\mathrm{Pr}_{0.5} \mathrm{Ca}_{1.5} \mathrm{MnO}_{4}$ films on $\mathrm{Nb}: \mathrm{SrTiO}_{3}$ with a charge-ordering transition at $T_{\mathrm{CO}} \sim 320 \mathrm{~K}$. This opens the route to a different phonon-bottleneck strategy of slowing down carrier relaxation by strong coupling of electrons to cooperative lattice modes.

DOI: 10.1103/PhysRevApplied.14.054006

\section{INTRODUCTION}

Establishing new mechanisms that overcome the constraints for photovoltaic energy conversion of conventional semiconductors due to transmission and thermalization losses is a high-priority goal of third-generation photovoltaics, for example, highly efficient solar cells that are based on thin films $[1,2]$. A primary goal to achieve higher efficiency is to overcome the Shockley-Queisser limit in semiconductors [3]. It arises from transmission losses of photons with energy below the bandgap as well as thermalization losses of hot carriers excited above the bandgap. The harvesting of hot carriers that normally undergo fast subpicosecond thermalization by scattering on optical phonons would be a major step into that direction. The

\footnotetext{
*cjooss@gwdg.de
}

Published by the American Physical Society under the terms of the Creative Commons Attribution 4.0 International license. Further distribution of this work must maintain attribution to the author(s) and the published article's title, journal citation, and DOI. concept of a phonon bottleneck as a strategy to slow down the thermalization of hot carriers has been proposed [4,5], i.e., carriers that are excited above the lower edge of the valence band. The phonon-bottleneck strategy aims for a reduction of energy loss of hot carriers via scattering on optical phonons, and subsequent dissipation and transport of the energy. It was successfully established in semiconductor quantum-dot systems [6,7]. Surprisingly, in some of these systems the excited-state stabilization of hot carriers occurred via strong coupling of coherent electron and phonon states in a confined geometry [8] rather than by a simple reduction of the phonon scattering.

Such strong coupling can result in hot polarons with lifetimes of tenths of picoseconds [6]. The hot-polaron lifetime is limited only by the stability of the electronphonon coupling in the excited state as well as the lifetime of the constituting particles, the electron, and the optical phonon. Therefore, in the absence of dipole relaxation that reduces the lifetime of the charge and anharmonicity that reduces the lifetime of the phonon, the hot-polaron state would be a long-lasting state that can be harvested by extraction of the hot carriers via an interface. It was recently shown that such a phonon-bottleneck 
strategy via strong electron-phonon coupling can be generalized to other material systems such as high-performance halide-perovskite solar cells that show polaron quasiparticles with long lifetime of hundreds of picoseconds [9-11].

Perovskite oxide thin films are an emerging system for next-generation solar cells due to strong and broad-band infrared-to-visible photon absorption and high stability in photoelectrochemical applications [12]. However, the slowdown of Mn $3 d e_{g}$-intraband carrier cooling by formation of small polarons is typically restricted to low temperatures and defect trapping [13]. In doped manganite perovskites, small Jahn-Teller (JT) and Zener polarons can be formed in the strong coupling regime [14-16]. Here, a dipole-allowed polaron excitation suitable for light harvesting exists within the $\mathrm{Mn} 3 d e_{g}-\mathrm{O} 2 p$ bands, which are split due to the JT polaron formation $[17,18]$. In an isolated $\mathrm{MnO}_{6}$ octahedron, the excitation of an electron from the occupied lower to the unoccupied higher JT split state relaxes through a conical intersection of a Mexican-hat-like energy potential surface (EPS) in configuration space within the period of a JT mode, i.e., $70 \mathrm{fs}$ [19]. This EPS represents the electron energy as a function of the displacement of the atoms. In contrast to the excitations in a single JT split $\mathrm{MnO}_{6}$ octahedron, the JT excitations in a three-dimensional (3D) periodic lattice of $\mathrm{MnO}_{6}$ octahedra exhibit a complex cooperative JT polaron and orbital dynamics, which can develop long-range correlations in the charge-ordered (CO) and orbital-ordered (OO) state [20]. We suggest that these long-range correlations of phonon modes involved in the polaron state represent an alternative type of phonon bottleneck. They can modify the EPS and thus can prevent the rapid structural relaxation through the conical intersection by local Jahn-Teller dynamics [19]. This enables polaron Mn $3 d$ $e_{g}$-intraband photovoltaic response as demonstrated for $\mathrm{Pr}_{0.65} \mathrm{Ca}_{0.35} \mathrm{MnO}_{3}$ with a charge- and orbital-order transition temperature $T_{\mathrm{CO}} \approx 240 \mathrm{~K}[18,21]$.

In this work, we demonstrate that harvesting of hotpolaron excitations in a charge-ordered small polaron system can be extended to room temperature and thus could provide a general concept for future photovoltaics. Via advanced material design we succeed in the epitaxial growth of two-dimensional (2D) layered RuddlesdenPopper manganite $\mathrm{Pr}_{0.5} \mathrm{Ca}_{1.5} \mathrm{MnO}_{4}$ (RP PCMO) thin films with $T_{c}$ well above room temperature. The high crystal quality and the coherence of the interface to the $\mathrm{Nb}$-doped $\mathrm{SrTiO}_{3}$ (STNO) single crystal is demonstrated using XRD and TEM. Optical spectroscopy and electrical transport reveal the expected polaron hopping, JT, and charge-transfer transitions at $T_{\mathrm{CO}} \approx 320 \mathrm{~K}$. The presence of a photovoltaic effect by hot-polaron excitations is demonstrated by the temperature-dependent measurement of current-voltage $(I-U)$ characteristics in different spectral ranges.

\section{EXPERIMENTAL METHODS}

\section{A. Film preparation by means of metalorganic aerosol deposition (MAD)}

Films with thicknesses of 30 to $200 \mathrm{~nm}$ are grown on [011]-oriented STO and STNO substrates using the metalorganic aerosol deposition technique. It is based on the heterogeneous pyrolysis of sprayed liquid solution, which contains a mixture of metalorganic precursors taken in appropriate molar relations. The deposition setup is equipped with an optical pyrometer and ellipsometry for in situ growth monitoring. The precursors, acetylacetonates of $\mathrm{Mn}, \mathrm{Pr}$, and $\mathrm{Ca}$, are dissolved in dimethylformamide to a concentration of 0.02 to $0.04 \mathrm{~mol} / \mathrm{l}$. The molar ratio between precursors in the solution as well as the growth conditions, $T_{\text {sub }}=800{ }^{\circ} \mathrm{C}$ and deposition rate approximately equal to $0.5 \mathrm{~nm} / \mathrm{s}$ are thoroughly optimized to allow the synthesis of material with a desired composition and structure.

\section{B. Film preparation by means of ion-beam sputtering} (IBS)

For the optical characterization a thicker epitaxial film of $100 \mathrm{~nm}$ thickness is prepared by means of ion-beam sputtering from single target on a single-crystal doubleside-polished STO [011] substrate. The deposition parameters for the film are set to $p_{\mathrm{Ar}}=3 \times 10^{-4}$ mbar (beam neutralizer), $p_{\mathrm{Xe}}=1 \times 10^{-4}$ mbar (sputter gas) and $p_{\mathrm{O} 2}=1.4$ $\times 10^{-4}$ mbar (film oxidation) The temperature of the boron nitride heater is set to $T=790{ }^{\circ} \mathrm{C}$, which corresponds to a substrate surface temperature of approximately $T_{S}=700{ }^{\circ} \mathrm{C}$. The cooling rate after deposition is $10^{\circ} \mathrm{C} / \mathrm{min}$ with 20 min holding steps at $690,490,290{ }^{\circ} \mathrm{C}$, respectively.

\section{Transmission electron microscopy}

The TEM lamellae in cross-section geometry are prepared in an FEI Nova NanoLab Dual Beam focused ionbeam system and thinned to electron transparency using $5 \mathrm{kV}$ during the final step. The subsequent TEM measurements are carried out in an FEI Titan 80-300 operated at $300 \mathrm{kV}$ and equipped with a Gatan Quantum 965 ER image filter. For the acquisition of selective area electron diffraction (SAED) patterns, a $10-\mu \mathrm{m}$ aperture is used, which translates to approximately $170 \mathrm{~nm}$ in the image plane. STEM images are taken at $42 \mathrm{pA}$ with an annular dark-field (ADF) detector having an inner and outer acceptance semiangle of 46.8 and $200 \mathrm{mrad}$ at the given camera length of $38 \mathrm{~mm}$. For the electron energy-loss spectroscopy (EELS) data acquisition with a collection semiangle of $39 \mathrm{mrad}$, the beam current is increased to $150 \mathrm{pA}$ and the resulting signals are analyzed using the power-law background subtraction routine of HyperSpy v1.4.2. [22]. 


\section{Electric characterization}

The four-probe in-plane electrical resistance of $\mathrm{RP}$ PCMO films is measured on insulating [011] STO substrates with silver-paste top contacts. The measurements are performed in a PPMS setup in the temperature range of $T=150-400 \mathrm{~K}$ and applied magnetic fields $B=0-9 \mathrm{~T}$.

\section{E. Optical characterization}

Optical spectroscopy is performed in a transmission and reflection setup in a UV-vis range of wavelength, $\lambda=200-1100 \mathrm{~nm}$ and in transmission in the NIR range, $\lambda=900-2500 \mathrm{~nm}$. In the UV-vis range the setup consists of an "OceanOptics DH-2000" Halogen light source, a fiberglass QP400-2-SR-BX, and Maya2000Pro spectrometer. The NIR setup includes a light source HL-2000-FHSA, a fiber QP400-2-SR-BX, and a NIR Quest512-2.5 spectrometer. For all measurements, a dark spectrum is subtracted by the equipment software. The transmittance is calculated by $I=I_{T} / I_{0}$, where $I_{0}$ is the incident and $I_{T}$ is the transmitted spectrally resolved intensity. This baseline correction eliminates contributions from the sample holder and mirror system. A fixed reflectance value of $R_{0}=0.3$ is assumed over the entire spectral range (for additional details see the Supplemental Material [23]).

\section{RESULTS AND DISCUSSION}

\section{A. Epitaxial growth of Ruddleson-Popper PCMO}

The orthorhombic crystal structure of RP PCMO is comprised of a perovskite layer $A \mathrm{MnO}_{3}(A=\mathrm{Pr}, \mathrm{Ca})$ separated by a rock-salt-layer $A \mathrm{O}$ (see inset Fig. 1), yielding a $2 \mathrm{D}$ network of $\mathrm{MnO}_{6}$ octahedra. The RP PCMO belongs to the space group Fmm 2 with $a=0.5365 \mathrm{~nm}, b=0.5354 \mathrm{~nm}$, and $c=1.1840 \mathrm{~nm}$ [24]. We prepare high-quality epitaxial heterojunctions of RP PCMO/ $\mathrm{SrTi}_{0.995} \mathrm{Nb}_{0.005} \mathrm{O}_{3}$ (STNO) with thicknesses between 30 and $200 \mathrm{~nm}$ grown by means of MAD [25,26] on (011) STNO substrates. In addition, a comparative study by ion-beam sputtering is pursued (for details see the Supplemental Material [23]). All films grown on (011) STNO substrates exhibit an orthorhombic structure with of $[100]_{\mathrm{RP}}$, respectively, $[010]_{\mathrm{RP}} \|[011]_{\mathrm{STO}}$ and in-plane alignment of their $c$ axis $[001]_{\mathrm{RP}} \|[100]_{\mathrm{STO}}$.

The out-of-plane epitaxial relation is confirmed by XRD (Fig. 1). The experimentally observed $(200)_{\mathrm{RP}} /(020)_{\mathrm{RP}}$ peak corresponds to a lattice spacing of $d_{h k l}=0.268 \mathrm{~nm}$, indicating a very small out-of-plane strain, despite the expected large tensile strain of $3 \%$ due to the lattice mismatch between RP PCMO and STNO. In addition, the Laue fringes around the $(200)_{\mathrm{RP}} /(020)_{\mathrm{RP}}$ peak imply uniform lattice spacing within the whole film. Hence, the films are fully relaxed.

In order to get insight into the microstructure of the RP films, STEM is performed on cross-section lamellas prepared along the [01 $\overline{1}]$ and [100] directions of the STNO

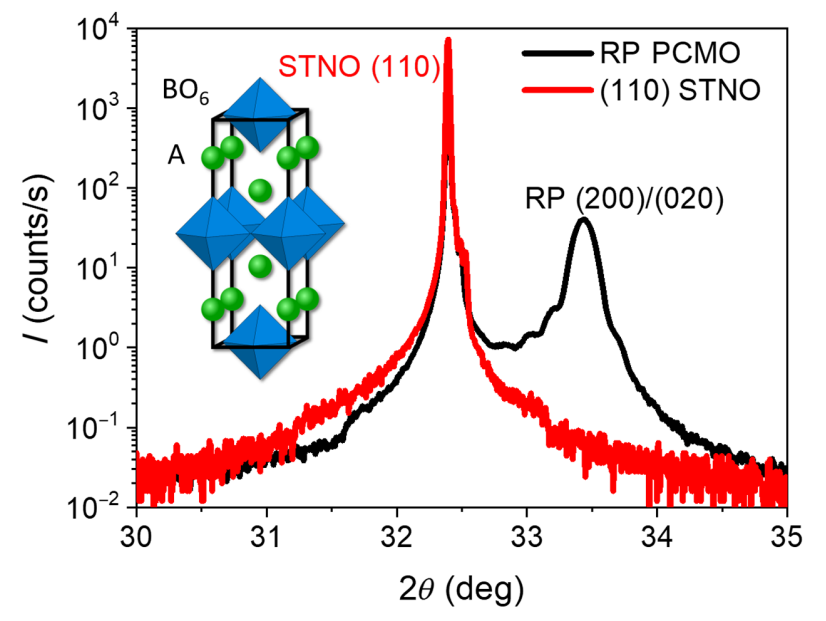

FIG. 1. XRD patterns of a 30-nm-thick MAD RP PCMO film on (011) STO and a blank substrate. Inset: pseudotetragonal representation of the $\mathrm{RP}\left(\mathrm{AA}^{\prime} \mathrm{BO}_{3}\right)_{1}\left(\mathrm{AA}^{\prime} \mathrm{O}\right)_{1}$ structure.

substrate, respectively. Figure 2(a) shows a high-resolution annular dark-field (ADF) STEM image of the lamella in the $[01 \overline{1}]$ zone axis, confirming the $c$-axis alignment along [100 $]_{\text {STO }}$ and the formation of a coherent manganitetitanite interface. Furthermore, a rather high density of planar defects aligned perpendicular to the substrate is visible, which appear at about $5 \mathrm{~nm}$ above the interface. High-resolution ADF imaging [Fig. 2(b)] provides evidence that the planar defects along the $c$ axis are stacking faults, a typical defect in RP phases. Such planar defects are not visible perpendicular to the $c$ axis, where misfit dislocations dominate.

Selective area electron diffraction confirms that the $c$ axis of the RP PCMO is aligned along the [100] in-plane direction of STNO [Fig. 2(d)]. Note that the out-of-plane reflections of STO and PCMO overlap. Furthermore, a discrimination between the RP PCMO [100] and RP PCMO [010] orientations along the growth direction cannot be made due to the small difference of the $a$ - and $b$-lattice parameters. In the in-plane direction, the STNO (001) and RP PCMO (002) reflections can be distinguished. The blurring of the RP PCMO (002) diffraction spot indicates the presence of disorder in this direction. A high density of planar defects along the $c$ direction is indeed clearly visible in the STEM image shown in Fig. 2(a).

Such planar defects are not observed perpendicular to the RP $c$ axis. High-resolution ADF STEM imaging of the interface along the STO [011] direction shows a semicoherent interface with misfit dislocations (Fig. 3).

The bright contrast close to the interface in Fig. 2(a) suggests a deviation in chemical composition within the first $5 \mathrm{~nm}$. In fact, high-resolution chemical analysis across the interface by means of electron energy-loss spectroscopy (EELS) reveals an increased $\mathrm{Pr}$ and a reduced Ca content in this region [Fig. 2(c)]. The Pr enrichment is associated with 


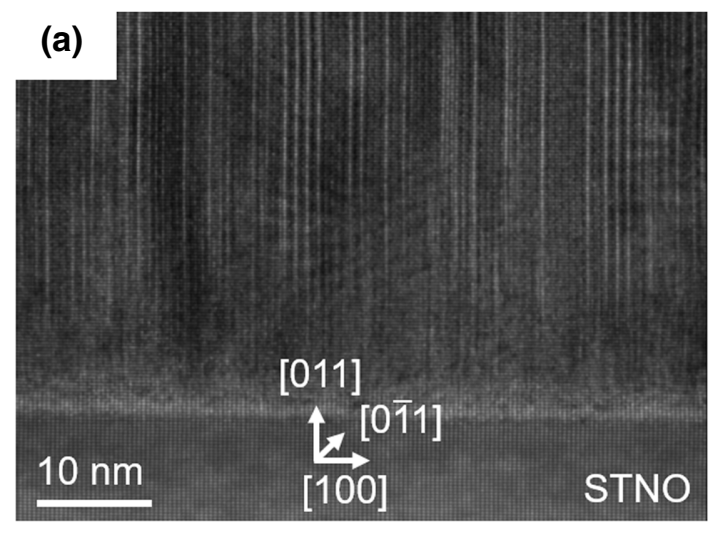

(c)

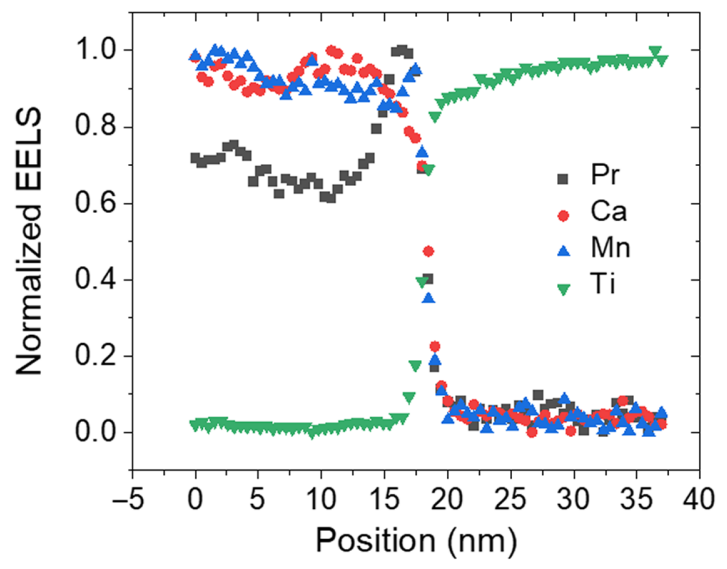

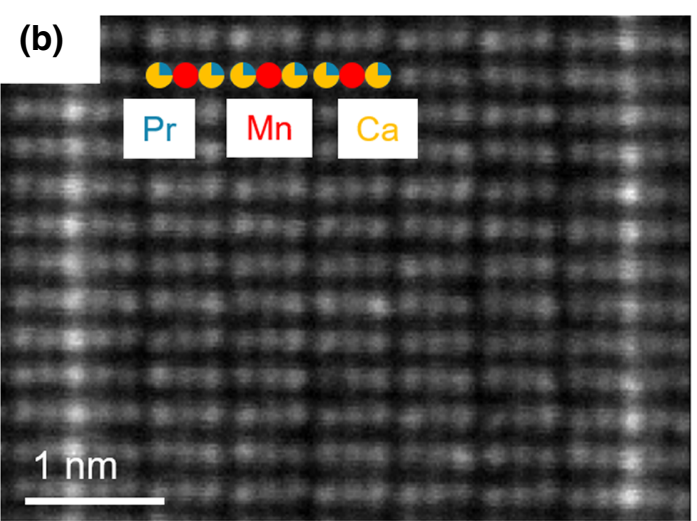

(d)
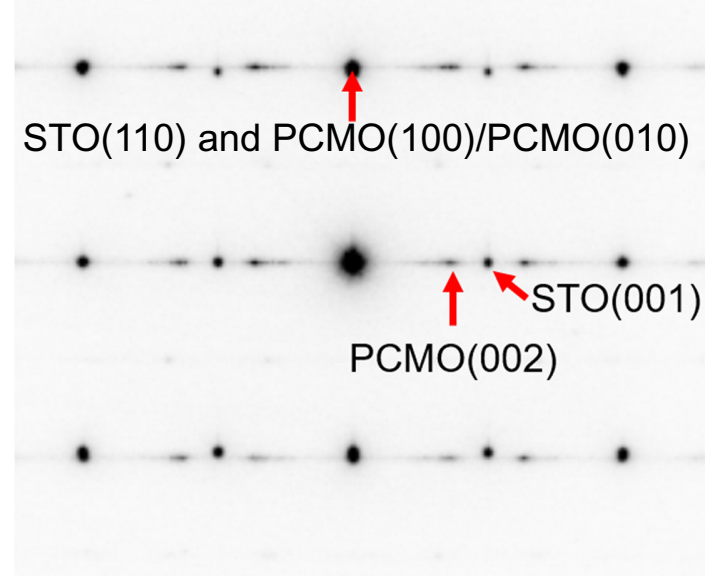

FIG. 2. Cross-section TEM imaging of RP PCMO on (011) STNO. The TEM lamella is prepared by focused ion-beam etching with the [011] zone axis of the STNO substrate. (a) High-resolution ADF STEM image of the film. (b) Enlarged picture of an area in (a). (c) Profile of the integrated EELS counts of the Ca, Ti, and Mn L as well as Pr M edge after power-law background subtractions normalized by the maximum. The concentration profiles across the interface between RP PCMO and STNO shows Pr segregation close to the interface. The RP layer sequence is visualized by the colored column markers. (d) Selective area electron diffraction pattern in the STO [011] zone axis shows reflections from the RP PCMO film and the STNO substrate due to the finite size of the SAED aperture.

an increase of the lattice parameters [27], suggesting that it represents an efficient misfit strain-relaxation mechanism that enables the growth of essentially strain-free films.

\section{B. Transport and optical properties}

The epitaxial RP PCMO films exhibit a remarkably high charge-ordering transition temperature of $T_{\mathrm{CO}}=320 \mathrm{~K}$ well above room temperature that agrees well with the $T_{\mathrm{CO}}$ of single crystals [26]. The $\mathrm{CO}$ transition is deduced from the changes in the temperature dependence of the in-plane electric resistivity, $\rho(T)$, measured along the $c$ axis of RP PCMO films grown on insulating STO (011) substrates. The exponential increase with decreasing temperature is consistent with thermally activated hopping mobility of small polarons [28] in the adiabatic limit:

$$
\rho(T)=\rho_{0} T e^{\frac{E_{\text {act }}}{k T}}
$$

Here, $T$ denotes temperature and $k$ the Boltzmann constant. In the disordered phase $\left(T>T_{\mathrm{CO}}\right), \rho(T) / T$ obeys an Arrhenius-type behavior with constant prefactor $\rho_{0}$ and activation energy $E_{\text {act }}$. The activation energy is modified in the charge-ordered phase, which allows for extracting $T_{\mathrm{CO}}$ from $\rho(T)$. Figure 4(a) shows the associated deviation of the measured resistance $\left(R_{\text {meas }}-R_{\mathrm{DO}}\right) / R_{\mathrm{DO}}$, where $R_{\text {DO }}$ represents the Arrhenius fit of Eq. (1) in the disordered (DO) phase, i.e., for $T>T_{\mathrm{CO}}$. This yields a polaron activation energy $E_{\text {act,DO }} \approx 163 \mathrm{meV}$ in the disordered phase. The inset of Fig. 4(a) depicts the changes in the temperature dependent activation energy by the phase transition obtained by differentiation of the data with respect to inverse temperature (see Ref. [29] for details). As expected, a pronounced increase of $E_{\text {act }}(T)$ in the chargeordered phase $\left(T<T_{\mathrm{CO}} \approx 320 \mathrm{~K}\right)$ is observed in complete (and even quantitative) agreement to 3D bulk manganites [29]. As a noteworthy secondary result, Fig. 4(a) further 


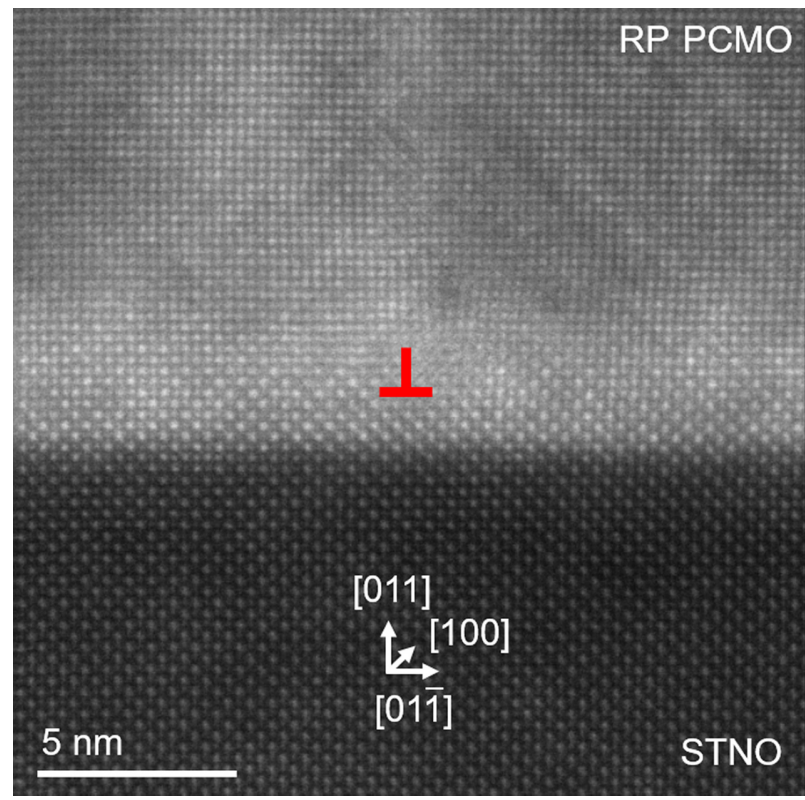

FIG. 3. High-resolution ADF STEM image of the interface of MAD grown RP PCMO on STO in the STO [100] zone axis. A misfit dislocation is marked by the red symbol.

shows that $T_{\mathrm{CO}}$ is independent of film thickness (compare data of the 200-nm-thick film), whereas the increase of $E_{\text {act }}$ in the charge-ordered state is smaller for the smaller thickness. The latter is attributed to a higher amount of disorder and residual strain. Nevertheless, the observed increase of the activation energy by about $20-30 \mathrm{meV}$ for the thin film is consistent with results obtained by Ibarra et al. in polycrystallite RP manganites $(0.5 \leq x \leq 0.8)$ [26].

Optical absorption measurements in Fig. 4(b) show the dominant contribution of small polaron absorption in the infrared and visible spectral range $0.6 \mathrm{eV} \leq \mathrm{h} \omega \leq 2.1 \mathrm{eV}$ and charge-transfer transitions for $\mathrm{h} \omega \geq 2.1 \mathrm{eV}$, in qualitative agreement with spectra of single crystals [30]. The presented spectrum can be well approximated by three Gaussian peaks located at $E_{A}=0.76 \mathrm{eV}, E_{B}=1.3 \mathrm{eV}$, and $E_{C}=3.5 \mathrm{eV}$. An intensity decrease of the Gaussian peak $A$ in the fitted absorption spectra below a limit value of $1 / e$ yields an estimated charge gap of $E_{g} \approx 0.57 \mathrm{eV}$.

In the following, processes related to the three absorption bands $A, B$, and $C$ are identified, which is relevant for the interpretation of photovoltaic effects in the system (see Fig. 5). According to the detailed study of 3D PCMO by Mildner et al. [18], we assign peak $A$ to an optically induced polaron hopping. As is well described by the theory of small polaron absorption [31], such an absorption band will occur at a photon energy $\mathrm{h} \omega$ double of the polaron formation energy $E_{p}$ and thus $E_{p}=$ $1 / 2 E_{A} \approx 380 \mathrm{meV}$. A completely different route is to estimate $E_{p}$ from the activation energy $E_{\text {act }}$ of thermally assisted hopping based on the relation $E_{\text {act }}=1 / 2 E_{P}-$
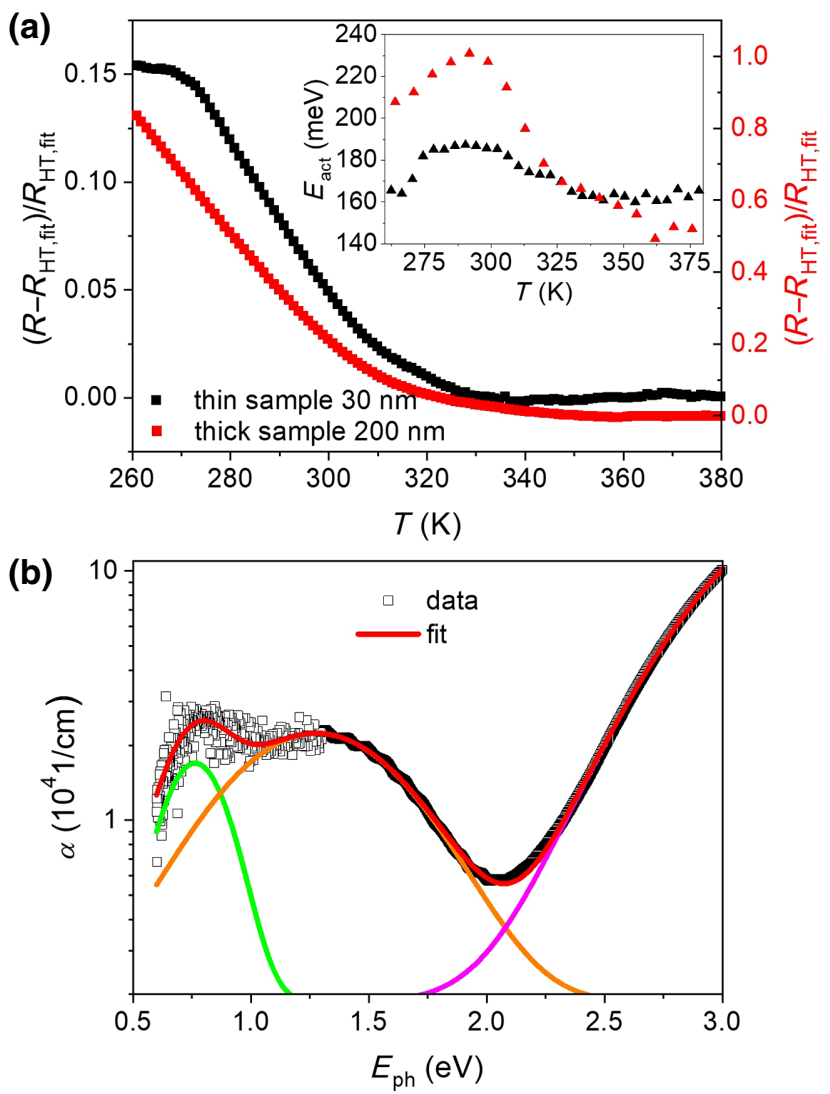

FIG. 4. Transport and optical properties of epitaxial [100]/[010] RP PCMO films on (011) STO. (a) Temperaturedependent deviation of resistance from the HT Arrhenius behavior and obtained temperature dependence of the activation energy (inset) for 30- and 200-nm-thick films. The change in slopes indicates the charge-ordering temperature of $T_{\mathrm{CO}} \approx 320 \mathrm{~K}$. (b) Spectrum of the absorption coefficient $\alpha$, measured for a 100-nm-thick RP PCMO film at room temperature. The experimental data is fitted by three Gaussian peaks, with $E_{A}=0.76 \mathrm{eV}$ (optical polaron hopping), $E_{B}=1.3 \mathrm{eV}$ (Jahn-Teller transition), and $E_{C}=3.5 \mathrm{eV}$ (charge-transfer transitions). The discontinuity in signal-to-noise ratio below $1.3 \mathrm{eV}$ stems from different spectrometers used for the UV and NIR regimes.

$J$. It can, e.g., be derived within the two-site Holstein model [32] and reflects that elastic tunneling between nextneighbor sites requires an isoenergetic configuration of the occupied and unoccupied site. Neglecting the contribution from the transfer integral $J, E_{\text {act }} \approx 190 \mathrm{meV}$ in the charge-ordered phase implies $E_{p} \approx 380 \mathrm{meV}$. The striking agreement of $E_{p}$ derived from electric transport and optical absorption strongly supports optically induced polaron hopping as the underlying microscopic process of absorption band $\mathrm{A}$. We note here, that the rather small contributions of band A to the photovoltaic cannot be detected within the sensitivity of our photovoltaic setup. Nevertheless, these infrared excitations can induce pronounced 


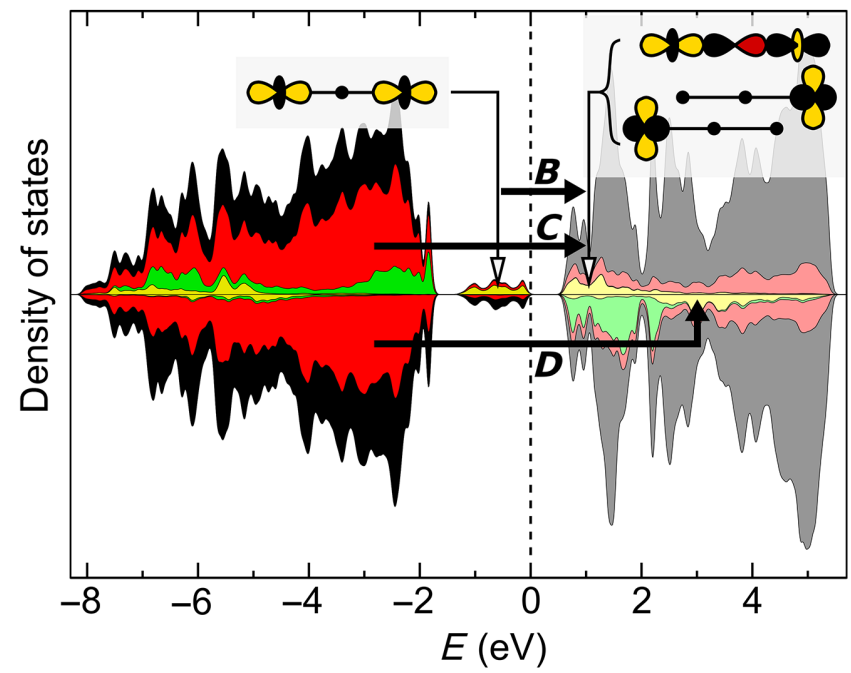

FIG. 5. Density of states of Ruddlesden-Popper $\operatorname{Pr}_{0.5} \mathrm{Ca}_{1.5} \mathrm{MnO}_{4}$ obtained from density-functional calculations (for details see Supplemental Material [23]). Majority and minority spin directions are shown with positive and negative sign. The colors correspond to the total density of states (black) and projections onto $\mathrm{O} p$ (red), as well as $\mathrm{Mn} t_{2 g}$ (green) and Mn $e_{g}$ (yellow) orbitals of the spin-up manganese atoms. The projected densities of states are shown stacked on top of each other; their values correspond to their colored areas. The JT splitting of the Mn $e_{g}$ states leads to an occupied nonbonding and three unoccupied states of $\sigma$ and $\delta$ character. Small polaron absorption is due to the dipole-allowed optical transition within the $\mathrm{Mn} d$ shell (peak $B$ ) from the nonbonding to antibonding $\sigma$ states. The dipole-allowed charge-transfer transitions (peaks $C$ and $D$ ) take place from nonbonding $\mathrm{O} 2 p$ states to majority and minority spin $\mathrm{Mn} e_{g}$ states, respectively.

photoenhanced mobility of polarons [33] and thus reduce Ohmic losses.

The absorption band in the UV regime (peak $C$ ) has two contributions, i.e., interband transitions in STO (above $3.2 \mathrm{eV}$ ) and charge-transfer (CT) transitions in PCMO that are dipole-allowed transitions between nonbonding $\mathrm{O} 2 p$ states and majority-spin $\mathrm{Mn} 3 d e_{g}$ states (see Fig. 5). Also such transitions have polaronic character, since the generated $\mathrm{O} 2 p$ holes and excited $\mathrm{Mn} 3 d$ majority spin electrons strongly couple to Jahn-Teller phonons in addition to other phonon modes.

Finally, the key for the hot-polaron photovoltaics reported in this work is photon absorption by band B in the NIR regime. It originates from transitions between $\mathrm{Mn} 3 d$ $e_{g}-\mathrm{O} 2 p$ states that are split due to the Jahn-Teller polaron formation, i.e., they are Mn $3 d e_{g}$-intraband transitions. They are dipole allowed due to their different parity as a result of the hybridization with $\mathrm{O} 2 p$ orbitals (see Fig. 5). In agreement with 3D PCMO, these states consist of nonbonding and antibonding Jahn-Teller split $e_{g}$ states of $\sigma$-bond character. Their origin is the hybridization of $\mathrm{Mn}$ $3 d e_{g}$ and $\mathrm{O} 2 p$ states at half doping that can be described as a Zener polaron, i.e., two coupled $\mathrm{MnO}_{6}$ octahedra [19]. The $3 \mathrm{D}$ or $2 \mathrm{D}$ topology of the $\mathrm{MnO}_{6}$ network only has a minor effect on the resulting DOS. Interestingly, in comparison to 3D perovskites and to the results of Ref. [29], we observe a reduced spectral weight resulting in a larger absorption length of the order of $\alpha\left(E_{B}\right)^{-1} \approx 400 \mathrm{~nm}$. This can be attributed to the anisotropic optical properties of the material and the epitaxial growth described above, which forces the $c$ axis being perpendicular to the incoming light at normal incidence. Before proceeding to the photovoltaic results, it should be emphasized that in contrast to the CT interband transition (absorption band C) typically observed in metal oxides, the polaron transitions of peaks $A$ and $B$ are stemming from $\mathrm{Mn} 3 d e_{g}$-intraband excitations that establish a pathway of harvesting hot-polaron states.

\section{Temperature-dependent photovoltaic response}

We show that charge ordering in the ground state is in fact a prerequisite for harvesting hot Jahn-Teller polarons from the Mn $3 d e_{g}$-intraband transitions of absorption B. In order to characterize the RP PCMO-STNO $p$-n heterojunctions current density-voltage $(J-U)$ measurements obtained in the dark at different temperature are summarized in Fig. 4(a). They exhibit rectifying characteristics with decreasing reverse-bias current and a shift of their exponential part to higher voltages by decreasing temperature. This behavior is completely in line with 3D PCMO and STNO junctions [6,21]. Within the one-diode model this behavior can be attributed to the increase of the diode ideality factor as well as of the shunt resistance, whereas contributions from series resistance to the total resistance is negligibly small.

Polychromatic illumination with a Xe-UV lamp leads to a photovoltaic effect with a downwards shift of the $J(U)$ curve [Fig. 6(b)]. According to the absorption bands described above, two main spectral ranges are distinguished: (i) charge-transfer interband excitations at photon energies of 2.2-4 eV, and (ii) low-energy $\mathrm{Mn} 3 d e_{g}$ intraband excitations for $0.5-2.2 \mathrm{eV}$ due to photoinduced hopping and Jahn-Teller-like excitations of small polarons. To separate these excitation processes, Fig. 6(b) shows the photovoltaic effect for a cut-off filter of $2.0 \mathrm{eV}$ where the photocurrent results from $\mathrm{A}$ and $\mathrm{B}$ absorption bands of the polaron excitations.

For a concise presentation of the temperature dependence, we use the open-circuit voltage $U_{\mathrm{OC}}$ for different spectral ranges [22] as shown in Fig. 6(c) for the RP PCMO thin films and-for comparison-for 3D PCMO perovskites. In addition to a full polychromatic illumination by the Xe lamp, the maximum excitation energy is limited by introducing different cut-off filters. The contribution of excitations at $\mathrm{h} \omega \leq 1.6 \mathrm{eV}$ is rather small at room temperature but it increases considerably below $230 \mathrm{~K}$. These excitations involve the optical polaron hopping 

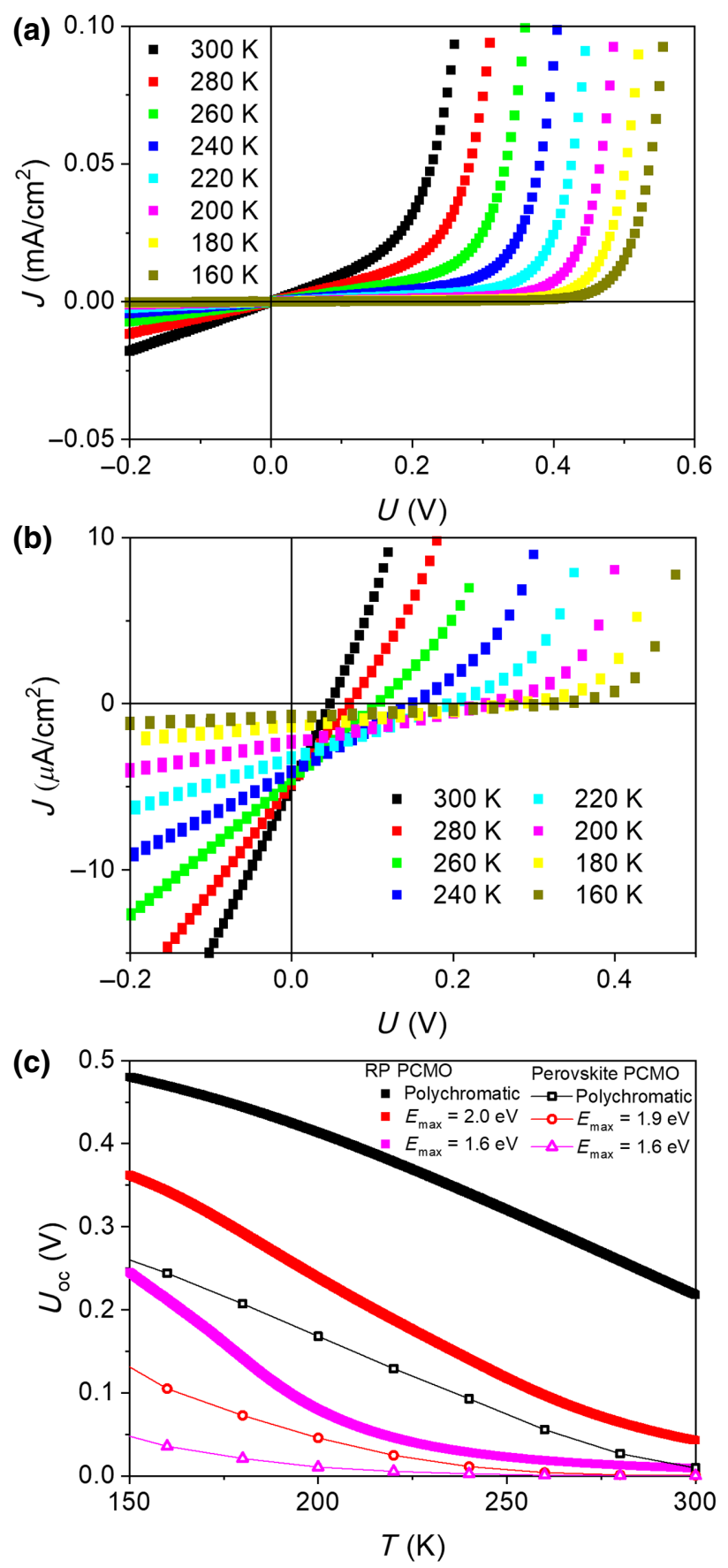

FIG. 6. Electric and photovoltaic characteristics of RP PCMO and STNO heterojunctions (a) Current density versus voltage as a function of temperature in the dark. (b) The same under illumination with low-pass filter for $\mathrm{h} \omega \leq 2.0 \mathrm{eV}$ photon energy. (c) Open-circuit voltage $U_{\mathrm{OC}}$ versus temperature for limited spectral illumination ranges. For comparison, data for heterojunctions of the $3 \mathrm{D}$ perovskite $\operatorname{Pr}_{0.66} \mathrm{Ca}_{0.34} \mathrm{MnO}_{3}$ on STNO is also included.

(peak $A$ ) as well as the major spectral weight of the JahnTeller transition (peak $B$ ). Only photon energies above the peak- $B$ maximum are able to excite polarons into a longliving state. Increasing the spectral range from $h \omega \leq 1.6 \mathrm{e}$ to $\mathrm{h} \omega \leq 2.0 \mathrm{eV}$ strongly impacts the photovoltaic effect at room temperature. The open-circuit voltage $U_{\mathrm{OC}}$ increases from 9 to $42 \mathrm{mV}$ due to the excitations of the upper shoulder of peak $B$. Above the peak maximum, the hot polaron forms a state where the $\mathrm{Mn} e_{g}$ population is inconsistent with its JT distortion and thus presumably underlies a long-range cooperative dynamics, similar to the one which has been observed in the 3D PCMO system [20] and is theoretically described by Rajpurohit et al. [34].

Under polychromatic illumination $U_{\mathrm{OC}}$ increases to $220 \mathrm{mV}$ at $300 \mathrm{~K}$ mostly due to the charge-transfer transitions in peak $C$. Furthermore, at photon energies above $3.2 \mathrm{eV}$, one can expect a contribution of band-band excitations in STNO to the photovoltaic effect, with electron-hole pairs contributing both from the titanate and the manganite.

For comparison, $U_{\mathrm{OC}}(T)$ data of the $3 \mathrm{D}$ perovskite-based $\mathrm{Pr}_{0.66} \mathrm{Ca}_{0.34} \mathrm{MnO}_{3} / \mathrm{STNO}$ heterojunctions is included in Fig. 6(c) [21]. In the 3D perovskites significant photovoltaic contributions due to polaronic electron-hole pair excitations only occur below the charge-order temperature $T_{\mathrm{CO}} \approx 240 \mathrm{~K}$, where such excitations reach sufficiently long lifetimes [19]. For the RP PCMO and STNO system, $T_{\mathrm{CO}} \approx 320 \mathrm{~K}$ results in pronounced polaron contributions to the photovoltaic effect even at room temperature, as is shown in Fig. 6(c). Therefore, it can be concluded, that the underlying longer lifetime of $\mathrm{Mn} e_{g}$-intraband hotpolaron excitations with energies above the charge gap are emerging only in the charge- and orbital-ordered state.

\section{CONCLUSION}

In summary, a room-temperature polaron photovoltaic effect is demonstrated in high-quality junctions of epitaxial RP PCMO on (011) STNO with charge-ordering temperature of $T_{\mathrm{CO}}=320 \mathrm{~K}$. Although the individual properties of small polarons in the RP PCMO with formation energy of $E_{p}=0.38 \mathrm{eV}$ are more or less unchanged compared to the 3D PCMO ( $\left.E_{p}=0.36-0.38 \mathrm{eV}[18]\right)$, the photovoltaic effect is remarkably enhanced. This result provides evidence that going from a 3D to a quasi-2D network of $\mathrm{MnO}_{6}$ octahedra changes the polaronic interactions and leaves their intrinsic properties virtually unaffected. That emphasizes again the key feature of cooperative hot-polaron behavior for photovoltaic energy conversion. In fact, the observation of Mn $3 d e_{g}$-intraband polaron photovoltaics being linked to the charge- and orbital-ordered state underlines the role of cooperative octahedral dynamics for the stabilization of long-living hot polarons. The polaron relaxation time in the $\mathrm{CO}$ and $\mathrm{OO}$ state may depend on both amplitude as well as long-range coherence of the involved lattice dynamics and, thus, depend on the excitation intensity [19-21]. Our result of polaron photovoltaic effect in the CO and OO state of the RP PCMO systems together with previous results of low-temperature polaron photovoltaics in ordered states of 3D manganites [21] 
suggests a promising different type of "phonon-bottleneck" mechanism for slowing down the carrier thermalization. The emergence of a coherent lattice oscillation of the $\mathrm{CO}$ and $\mathrm{OO}$ superlattice reflections as observed by Beaud [35] may avoid the fast structural relaxation as expected for the incoherent dynamics of individual JT polarons. Our work demonstrates that room-temperature photovoltaic energy conversion in the visual to infrared spectral range in complex oxides with a small or vanishing band gap in the excitation spectrum by hot polarons is feasible. It moreover implies that material design along the route of improving excess carrier lifetime through involved phonon states that are strongly coupled to the charge carriers and display pronounced long-range coherence can serve as a blueprint to enhance the efficiency of hot-polaron photovoltaics.

\section{ACKNOWLEDGMENTS}

The authors thank K. Stroh for the help in optical spectroscopy measurements and the authors are thankful for funding by the Deutsche Forschungsgemeinschaft (DFG, German Research Foundation-217133147/SFB 1073, projects A02, B02, B03, Z02). The use of equipment in the "Collaborative Laboratory and User Facility for Electron Microscopy" (CLUE) is gratefully acknowledged.

[1] M. Green, Third Generation Photovoltaics (Springer, Berlin Heidelberg, 2003).

[2] M. A. Green and S. P. Bremner, Energy conversion approaches and materials for high-efficiency photovoltaics, Nat. Mater. 16, 23 (2017).

[3] W. Shockley and H. J. Queisser, Detailed balance limit of efficiency of p-n junction solar cells, J. Appl. Phys. 32, 510 (1961).

[4] H. Benisty, C. M. Sottomayor-Torres, and C. Weisbuch, Intrinsic mechanism for the poor luminescence properties of quantum-box systems, Phys. Rev. B 44, 10945 (1991).

[5] G. Conibeer, S. Shrestha, S. Huang, R. Patterson, H. Xia, Y. Feng, P. Zhang, N. Gupta, M. Tayebjee, S. Smyth, et al., Hot carrier solar cell absorber prerequisites and candidate material systems, Sol. Energy Mater. Sol. C. 135, 124 (2015).

[6] S. Sauvage, P. Boucaud, R. P. S. M. Lobo, F. Bras, G. Fishman, R. Prazeres, F. Glotin, J. M. Ortega, and J.-M. Gérard, Long Polaron Lifetime in InAs/GaAs Self-Assembled Quantum Dots, Dots Phys. Rev. Lett. 88, 177402 (2002).

[7] W. A. Tisdale, K. J. Williams, B. A. Timp, D. J. Norris, E. S. Aydil, and X.-Y. Zhu, Hot-Electron transfer from semiconductor nanocrystals, Science 328, 1543 (2010).

[8] S. Hameau, Y. Guldner, O. Verzelen, R. Ferreira, G. Bastard, J. Zeman, A. Lemaitre, and J. M. Gérard, Strong Electron-Phonon Coupling Regime in Quantum Dots: Evidence for Everlasting Resonant Polarons, Phys. Rev. Lett. 83, 4152 (1999).

[9] J. Moore Frost, L. D. Whalley, and A. Walsh, Slow cooling of Hot polarons in halide perovskite solar cells, ACS Energy Lett. 2, 2647 (2017).
[10] M. Park, A. J. Neukirch, S. E. Reyes-Lillo, M. Lai, S. R. Ellis, D. Dietze, J. B. Neaton, P. Yang, S. Tretiak, and R. A. Mathies, Excited-state vibrational dynamics toward the polaron in methylammonium lead iodide perovskite, Nat. Commun. 9, 2525 (2018).

[11] D. Ghosh, E. Welch, A. J. Neukirch, A. Zakhidov, and S. Tretiak, Polarons in halide perovskites: A perspective, J. Phys. Chem. Lett. 11, 3271 (2020).

[12] M. Lira-Cantu, The Future of Semiconductor Oxides in Next-Generation Solar Cells (Elsevier, Amsterdam, 2018).

[13] O. Bidault, M. Maglione, M. Actis, M. Kchikech, and B. Salce, Polaronic relaxation in perovskites, Phys. Rev. B 52, 4191 (1995).

[14] G. Zhao, K. Conder, H. Keller, and K. A. Müller, Giant oxygen isotope shift in the magnetoresistive perovskite $\mathrm{La}_{1-x} \mathrm{Ca}_{x} \mathrm{MnO}_{3+y}$, Nature 381, 676 (1996).

[15] G. Zhao, Y. S. Wang, D. J. Kang, W. Prellier, M. Rajeswari, H. Keller, T. Venkatesan, C. W. Chu, and R. L. Greene, Evidence for the immobile bipolaron formation in the paramagnetic state of the magnetoresistive manganites, Phys. Rev. B 62, R11 949 (2000).

[16] M. Sotoudeh, S. Rajpurohit, P. E. Blöchl, D. Mierwaldt, J. Norpoth, V. Roddatis, S. Mildner, B. Ifland, and C. Jooss, Electronic structure of $\mathrm{Pr}_{1-x} \mathrm{Ca}_{x} \mathrm{MnO}_{3}$, Phys. Rev. B 95, 235150 (2017)

[17] J. H. Jung, K. H. Kim, D. J. Eom, T. W. Noh, E. J. Choi, J. Yu, Y. S. Kwon, and Y. Chung, Determination of electronic band structures of $\mathrm{CaMnO}_{3}$ and $\mathrm{LaMnO}_{3}$ using optical-conductivity analyses, Phys. Rev. B 55, 15489 (1997).

[18] S. Mildner, J. Hoffmann, C. Jooss, P. E. Bloechl, and S. Techert, Temperature- and doping-dependent optical absorption in the small-polaron system $\operatorname{Pr}_{1-x} \mathrm{Ca}_{x} \mathrm{MnO}_{3}$, Phys. Rev B. 92, 035145 (2015).

[19] D. Raiser, S. Mildner, B. Ifland, M. Sotoudeh, P. Blöchl, S. Techert, and Ch. Jooss, Contribution of Jahn-Teller and charge transfer excitations to the photovoltaic effect of manganite/titanite heterojunctions, Adv. Energy Mater. 7, 1602174 (2017).

[20] P. Beaud, A. Caviezel, S. O. Mariager, L. Rettig, G. Ingold, C. Dornes, S.-W. Huang, J. A. Johnson, M. Radovic, T. Huber, et al., A time-dependent order parameter for ultrafast photoinduced phase transitions, Nat. Mater. 13, 923 (2014).

[21] B. Ifland, J. Hoffmann, B. Kressdorf, V. Roddatis, M. Seibt, and Ch. Jooss, Contribution of Jahn-Teller and charge transfer excitations to the photovoltaic effect of manganite/titanite heterojunctions, New J. Phys. 19, 063046 (2017)

[22] F. de la Peña, E. Prestat, V. T. Fauske, et al., 2019 hyperspy/hyperspy: v1.4.2.

[23] See Supplemental Material at http://link.aps.org/supplemen tal/10.1103/PhysRevApplied.14.054006 for a detailed description of electrical and optical characterization, density of state calculations, and additional x-ray diffraction and TEM studies of IBS-prepared thin films. Includes Refs. [16,36-40].

[24] M. Ebrahimizadeh Abrishami, M. Risch, J. Scholz, V. Roddatis, N. Osterthun, and C. Jooss, Oxygen evolution at manganite perovskite ruddlesden-popper type particles: Trends of activity on structure, valence and covalence, Materials 9, 921 (2016). 
[25] M. Jungbauer, S. Hühn, R. Egovail, H. Tan, J. Verbeeck, G. Tandeloo, and V. Moshnyaga, Atomic layer epitaxy of ruddlesden-popper $\mathrm{SrO}\left(\mathrm{SrTiO}_{3}\right)$ n films by means of metalorganic aerosol deposition, APL 105, 251603 (2014).

[26] A. Belenchuk, O. Shapoval, V. Roddatis, V. BruchmannBamberg, K. Samwer, and V. Moshnyaga, RuddlesdenPopper interface in correlated manganite heterostructures induces magnetic decoupling and dead layer reduction, APL 109, 232405 (2016).

[27] M. Ibarra, R. Retoux, M. Hervieu, C. Autret, A. Maignan, C. Martin, and B. Raveau, Charge-orbital ordering above room temperature in the $2 \mathrm{D} \mathrm{Pr}_{1-x} \mathrm{Ca}_{1+x} \mathrm{MnO}_{4}$ manganites, J. Solid State Chem. 170, 361 (2003).

[28] V. V. Bryksin and H. Böttger, Hopping Conduction in Solids, 1st ed. (VCH, Weinheim, 1985).

[29] J. Hoffmann, P. Moschkau, S. Mildner, J. Norpoth, Ch. Jooss, L. Wu, and Y. Zhu, Effects of interaction and disorder on polarons in colossal resistance manganite $\operatorname{Pr}_{0.68} \mathrm{Ca}_{0.32} \mathrm{MnO}_{3}$ thin films, Mater. Res. Express 1, 046403 (2014).

[30] M. A. Majidi, E. Thoeng, P. K. Gogoi, F. Wendt, S. H. Wang, I. Santoso, T. C. Asmara, I. P. Handayani, P. H. M. van Loosdrecht, A. A. Nugroho, et al., Temperaturedependent and anisotropic optical response of layered $\mathrm{Pr}_{0.5} \mathrm{Ca}_{1.5} \mathrm{MnO}_{4}$ probed by spectroscopic ellipsometry, Phys. Rev. B 87, 235135 (2013).

[31] D. Emin, Optical properties of large and small polarons and bipolarons, Phys. Rev. B 48, 13691 (1993).

[32] T. Holstein, Studies of polaron motion, studies of polaron motion: Part I. The molecular-crystal model, Ann. Phys. 8, 325 (1959).
[33] J. F. Xi, K. Zhao, H. Ni, W. F. Xiang, X. Feng, Q. Sun, Z. Q. Lu, and L. Z. Xiao, Giant negative photoresistance of epitaxialmanganite $\mathrm{La}_{2 / 3} \mathrm{Ca}_{1 / 3} \mathrm{MnO}_{3-\delta}$ film, Sci. China 58, 122402 (2015).

[34] S. Rajpurohit, Ch. Jooss, and P. E. Blöchl, Evolution of the magnetic and polaronic order of $\operatorname{Pr}_{1 / 2} \mathrm{Ca}_{1 / 2} \mathrm{MnO}_{3}$ following an ultrashort light pulse, Phys. Rev. B 102, 014302 (2020).

[35] P. Beaud, S. L. Johnson, E. Vorobeva, U. Staub, R. A. De Souza, C. J. Milne, Q. X. Jia, and G. Ingold, Ultrafast Structural Phase Transition Driven by Photoinduced Melting of Charge and Orbital Order, Phys. Rev. Lett. 103, 155702 (2009).

[36] I. P. Handayani, A. A. Nugroho, S. Riyadi, G. R. Blake, N. Mufti, T. T. M. Palstra1, and P. H. M. van Loosdrecht, Correlation between lattice vibrations with charge, orbital, and spin ordering in the layered manganite $\operatorname{Pr}_{0.5} \mathrm{Ca}_{1.5} \mathrm{MnO}_{4}$, Phys. Rev. B 92, 205101 (2015).

[37] P. E. Blöchl, Projector augmented-wave method, Phys. Rev. B 50, 17953 (1994).

[38] Peter E. Blöchl and Clemens Först, ,Node-less atomic wave functions, Pauli repulsion and systematic projector augmentation" arXiv:1210.5937 [physics. chem-ph].

[39] O. K. Andersen and O. Jepsen, Explicit, First-Principles Tight-Binding Theory, Phys. Rev. Lett. 53, 2571 (1984).

[40] E. O. Wollan and W. C. Koehler, Neutron diffraction study of the magnetic properties of the series of perovskitetype compounds $\left[{ }_{(1-x)} \mathrm{La}_{,} \mathrm{Ca}\right] \mathrm{MnO}_{3}$, Phys. Rev. 100, 545 (1955). 